\title{
Dual Microcatheter Retrograde Transvenous Obliteration of Gastric Varices: Coil Embolization as a Substitute for Balloon Occlusion
}

\author{
Hiroki Minamiguchi Nobuyuki Kawai Morio Sato \\ Munehisa Sawa Akira Ikoma Hiroki Sanda \\ Kouhei Nakata Motoki Nakai Tetsuo Sonomura \\ Department of Radiology, Wakayama Medical University, Wakayama, Japan
}

\section{Key Words}

Balloon-occluded retrograde transvenous obliteration - Gastric varices - Microcoil · Microcatheter

\begin{abstract}
Dual microcatheter retrograde transvenous obliteration (DMRTO) of gastric varices enables dual microcatheters to be advanced to the gastric varices themselves or to a site adjacent to the varices. The sclerosing agent is infused through the first microcatheter following coil embolization of the outflow vessels through the second microcatheter, which is placed several centimeters back from the varices. We present two cases of gastric varices in whom balloon-occluded retrograde transvenous obliteration failed, because of angulated gastrosubphrenic shunt in case 1 and a tortuous and elongated gastrorenal shunt in case 2 . DMRTO successfully achieved eradication of the gastric varices in both cases.
\end{abstract}

\section{Introduction}

Balloon-occluded retrograde transvenous obliteration (BRTO) has become a well-established treatment for gastric varices since the first report by Kanagawa et al. in 1996 [1]. The treatment theory is to infuse a sclerosing agent to the gastric varices under occlusion of the draining vessel by balloon inflation. Gastric varices commonly form a spontaneous gastrorenal (GR) shunt, with the left adrenal vein targeted as the main outflow vessel by balloon inflation. The success rate in obliteration of gastric varices was $70-93 \%$ of complete obliteration by a single BRTO procedure in the 
previous reports [2]. However, balloon-occluded left adrenal venography does not generally lead to delineation of the gastric varices, because of the presence of other outflows, such as the inferior phrenic vein, pericardial vein, cardiophrenic vein, and retroperitoneal vein [3-9]. In this case, the procedure can be performed using a variety of devices, such as coil embolization of small outflow vessels, additional sclerosant injection of 50\% glucose and lipiodol-ethanol for occlusion of outflow vessels, additional coaxial microballoon occlusion beyond the outflow vessels [10-15], and temporary balloon occlusion of the splenic artery [2]. Then, the possible situation of unsuccessful BRTO still remains.

We present two cases of gastric varices in whom treatment by BRTO failed, but who were treated by dual microcatheter retrograde transvenous obliteration (DMRTO) without balloon occlusion. The Institutional Review Board at our hospital did not require approval for this retrospective case study.

\section{Case Reports}

\section{Facility}

Each patient received DMRTO at an angio-CT facility (General Electric Medical Systems, Milwaukee, Wisc., USA). The angio-CT facility utilizes a common tabletop that enables angiography or computed tomography (CT) to be performed without transferring the patient.

\section{Case 1 (fig, 1)}

A 68-year-old man attended our hospital for prophylactic treatment of gastric varices revealed by gastroendoscopy at his local hospital. He had previously received endoscopic intravariceal sclerotherapy for esophageal varices and radiofrequency ablation for hepatocellular carcinoma (HCC). Laboratory tests revealed Child-Pugh A (score 6) liver cirrhosis caused by hepatitis B virus infection.

Multiplanar reconstruction (MPR) images of dynamic CT were obtained by contrast-enhanced multidetector row helical CT (MDCT) (LightSpeed VCT 64, General El ectric Healthcare, Milwaukee, Wisc., USA). The 3D-MPR images demonstrated gastric varices: the inflow vessel was the posterior gastric vein and the outflow vessel was a dilated subphrenic vein draining to the inferior vena cava, with no GR shunt. Referring to the coronal images of the MDCT, BRTO from the left subphrenic vein was attempted.

The right hepatic venous branch was catheterized and hepatic vein wedge pressure was measured before treatment using a $5 \mathrm{Fr}$ balloon catheter (C2 type, Selecon MP catheter, Terumo Clinical Supply, Gifu, Japan) through a C-shaped sheath (7 Fr, Medikit, Miyazaki, Japan) inserted from the right femoral vein. Wedge pressure was measured using a manometer (Polygraph, MSC-7000, Fukuda Denshi, Tokyo, Japan) after occluding the vein by inflating the balloon catheter with diluted contrast medium. The zero reference point for each measurement was set at the midaxillary line of the patient. Hepatic wedge pressure was $14 \mathrm{~mm} \mathrm{Hg}$ before treatment.

The left subphrenic vein was first catheterized using a 6.2 Fr Elway catheter (C2 type, Terumo Clinical Supply) as a guide catheter through an $8 \mathrm{Fr}$ sheath (Terumo Clinical Supply) via the left femoral vein. A microballoon catheter (3.3 Fr, Iiguman, temporary occlusion balloon C2 type, Fuji Systems, Fukushima, Japan) was then inserted coaxially through the guide catheter to the left subphrenic vein and microballoon-occluded left subphrenic venography was performed to demonstrate the subphrenic branch vein without the gastric varices. The microballoon catheter was further advanced proximally but could not be inserted into the subphrenic branch vein because of the abrupt angle. The microballoon catheter was then replaced with a microcatheter $(2.4 \mathrm{Fr}$, 
MicroFeret-18, William Cook, Bjaeverskov, Denmark) using a microguidewire (0.014 inch, Hi-Lex, Hyogo, Japan). $10 \mathrm{ml}$ of $50 \%$ glucose and $7.5 \mathrm{ml}$ of $5 \%$ ethanolamine oleate-iopamidol (EOI) were injected in vain to occlude the inferior phrenic branch vein. The microcatheter was inserted to the inferior phrenic branch vein, but extravasation of contrast medium occurred probably because of the thin-sized and abrupt-angled vein. Then, the microcatheter was advanced to the outflow vessel adjacent to the gastric varices through the subphrenic branch vein. Test injection via the microcatheter showed that blood flow from the inflow vessel was rapid. However, the site of the microcatheter was confirmed on test injection image with reference to preoperative 3D-MPR images. Repeat selective catheterization of the left subphrenic vein was conducted using the guide catheter already inserted into the right hepatic vein.

A new microcatheter (2.2 Fr, Shirabe, Piolax, Yokohama, Japan) was coaxially inserted through this guide catheter and advanced to the same site where the first microcatheter had been placed. The second microcatheter was pulled back several centimeters. Coil embolization of the outflow vessel was then performed through the second microcatheter and continued until stagnation of the contrast medium in the entire gastric varices was confirmed by test injection from the first microcatheter. The used microcoils through the second microcatheter were as follows: interlocking detachable coils, $8 \mathrm{~mm} / 10 \mathrm{~cm}, 1$ piece, $7 \mathrm{~mm} / 10 \mathrm{~cm}, 1$ piece (Boston Scientific, Cork, Ireland); Tornado embolization coils, $4 / 6 \mathrm{~mm}, 2$ pieces (William Cook). To reduce the dose of sclerosing agent, $20 \mathrm{ml}$ of $50 \%$ glucose was infused initially. As the sclerosing agent, 5\% EOI mixture was used, mixed with $10 \%$ ethanolamine oleate (Oldamin, Fuji Chemical, Toyama, Japan) and the same dose of contrast medium (iopamidol, $300 \mathrm{mg} / \mathrm{ml}$ ). Intravenous drip infusion of 4,000 units of human haptoglobin (Benesis, Osaka, Japan) was administered for approximately $30 \mathrm{~min}$ before treatment. $9 \mathrm{ml}$ of $5 \%$ EOI mixture as the same volume of contrast medium used to delineate the gastric varices was infused into the outflow vessel and gastric varices. CT was obtained immediately after treatment to confirm accumulation of the mixture in the gastric varices. After treatment, the hepatic vein was re-catheterized and hepatic vein wedge pressure was measured. Hepatic wedge pressure after treatment was $42 \mathrm{~mm} \mathrm{Hg}$. The dual catheter systems were withdrawn immediately after treatment.

Contrast-enhanced CT the day after treatment showed thrombosis of the gastric varices without ascites. No adverse effects except for transient hematuria and mild fever were observed. Laboratory tests after treatment revealed an improvement in Child-Pugh score from 9 to 8.

\section{Case 2 (fig. 2)}

A 63-year-old man attended our hospital having been diagnosed with gastric varices and HCC at his local hospital. Laboratory data revealed Child-Pugh C (score 11) liver cirrhosis caused by hepatitis C virus infection. Coronal MPR CT images demonstrated gastric varices with GR shunt composed of numerous tortuous inflow and outflow vessels, and a small HCC, $2 \mathrm{~cm}$ in diameter, in the left lobe. Following a multidisciplinary conference among surgeons, interventional radiologists and hepatologists, the patient was scheduled to receive BRTO for gastric varices, followed by transcatheter arterial embolization and radiofrequency ablation for HCC because rupture of the gastric varices was viewed to be more life-threatening than the growth of the HCC.

Catheter treatment for gastric varices was conducted. The right hepatic vein was catheterized via the right femoral vein to measure hepatic vein wedge pressure using the same sheath and balloon catheters as described for case 1 . Because catheterization of the left adrenal vein via the femoral vein was difficult, a 6 Fr balloon catheter (Selecon MP, Cobra, balloon diameter $20 \mathrm{~mm}$, Terumo, Tokyo, Japan) was inserted into the left adrenal vein through an 8 Fr sheath (Terumo Clinical Supply) via the right jugular vein. Despite coil embolization (Tornado $2 / 5 \mathrm{~mm}, 3$ pieces, William Cook) of outflow vessels and injection of $20 \mathrm{ml}$ of $50 \%$ glucose, $2 \mathrm{ml}$ of lipiodol-ethanol, and $7.5 \mathrm{ml}$ of $5 \%$ EOI, balloon-occluded left adrenal venography did not demonstrate the gastric varices. Further advancement of the balloon catheter toward the varices was difficult because of the markedly tortuous varicosity. Finally, a microcatheter $(2.4 \mathrm{Fr}$, MicroFeret-18) was advanced through the balloon catheter using a microwire ( 0.014 inch, Hi-Lex) to reach the gastric varices. The site of the microcatheter tip was confirmed on plain CT with reference to the preoperative 3D-MPR images.

We intended to insert another microcatheter into the gastric varices. A 5 Fr catheter (1CJ type, Terumo Clinical Supply) was inserted as a guide catheter into the left adrenal vein through a $6 \mathrm{Fr}$ 
sheath via the left femoral vein, and the second microcatheter (2.2 Fr, Shirabe, Piolax) was coaxially inserted through the guide catheter using a microwire ( 0.014 inch, Hi-Lex) and advanced to the same position as the first microcatheter. The rest of the procedure was almost the same as described for case 1: the microcatheter was pulled back several centimeters and coil embolization of the outflow vessel was conducted and continued through the second microcatheter until test injection through the first microcatheter delineated stagnation of contrast medium in the gastric varices. The used microcoils were as follows: interlocking detachable coils, $8 \mathrm{~mm}$ diameter, $10 \mathrm{~cm}$ long, 7 pieces (Boston Scientific). Intravenous drip infusion of 4,000 units of human haptoglobin (Benesis) was administered for approximately $30 \mathrm{~min}$ before treatment. The same volume $(15 \mathrm{ml})$ of $5 \%$ EOI as the volume of contrast medium used to delineate the gastric varices was infused into the outflow vessel and gastric varices. Immediately after treatment, CT was obtained to confirm accumulation of $5 \%$ EOI in the varices. After treatment, the hepatic vein was re-catheterized and hepatic vein wedge pressure was measured. Hepatic wedge pressure before and after treatment was 24 and $34 \mathrm{~mm} \mathrm{Hg}$, respectively. The triple catheter system was withdrawn immediately after treatment. Contrast-enhanced CT the following day revealed thrombosis of the gastric varices. No adverse effects except for transient hematuria and mild fever were observed. Laboratory tests revealed an improvement in Child-Pugh score from 11 to 10.

\section{Discussion}

Even when gastric varices are associated with GR shunt, they are commonly not delineated on balloon-occluded left adrenal venography because of the presence of other outflows [3-6, 12-14]. To accomplish BRT0, it is necessary to occlude these outflows so that the gastric varices and GR shunt form a single route; however, if the GR shunt is tortuous and elongated, it is difficult to embolize all of the small outflows. In this situation, the varices are not delineated because the contrast medium simply flows through these outflow routes into the vena cava. In case 2, we performed coil embolization and infusion of sclerosing agent of $50 \%$ glucose, lipiodol-ethanol, and small amounts of EOI to occlude the other outflows, but the gastric varices could not be delineated under balloon occlusion because of the tortuous and elongated GR shunt. Accordingly, DMRTO is a feasible and useful method for treating cases of gastric varices in whom the GR shunt is so tortuous and elongated that BRTO is unsuccessful.

When gastric varices are present without GR shunt, the subphrenic, pericardial, and paraesophageal veins are involved as outflow vessels instead of the left adrenal vein, and it can be difficult to achieve BRTO for eradication of the varices. In this situation, percutaneous transhepatic obliteration and endoscopic intravariceal sclerotherapy using cyanoacrylate glue can be performed instead of BRTO. Takahashi et al. reported the usefulness of a coaxial microballoon catheter system for selective sclerosis in the case of failure of BRTO [12]. However, in the present cases, the microballoon catheter system could not be used because of abrupt vessel angulations. The DMRTO method was used as an alternative procedure because its more flexible microcatheter could be advanced more proximally than a microballoon catheter. Accordingly, in the present case 1, DMRT0 was feasible and useful for treatment of gastric varices without GR shunt.

The first step in DMRTO of gastric varices is to advance the dual microcatheters peripherally as far as the gastric varices themselves, or to a site adjacent to the varices. The first microcatheter remains in place to infuse the sclerosing agent, while the second microcatheter is pulled back a few centimeters for coil embolization. Preoperative image analysis of the inflow vessels, gastric varices, and outflow vessels using MPR CT 
is crucial for DMRTO. To achieve DMRTO, it is important to confirm during the procedure whether or not the microcatheter is advanced to the gastric varices. The position of the microcatheter tip was confirmed by test injection in case 1 and observed on plain CT obtained during angio-CT adjacent to the gastric varices in case 2, referring to the preoperative enhanced MPR CT images in each. The preoperative MPR CT images are useful as a reference of the target site during the procedure.

Several weaknesses exist in the DMRTO method. The method is not independent of the BRTO method, and BRTO generally precedes DMRTO. Even when gastric varices cannot be delineated, the balloon-occluded venography images are indispensable for tracing the position of the dual microcatheters. In both of the present cases, the BRTO method was first attempted for gastric varices; when BRTO failed, DMRTO was used as an alternative procedure. The second weakness is that it is imperative to determine whether the microcatheter tip has reached the gastric varices. In the present cases, it was necessary to take the preoperative enhanced MPR CT images. In case of no enhanced MPR CT images, the venous phase of splenic arteriography might be an alternative imaging method for identifying the microcatheter tip. Third, it takes time to advance the dual microcatheters to the gastric varices or to a site adjacent to the varices._However, the second microcatheter catheterization to the same position where the first microcatheter was situated was not so difficult compared to the first microcatheter catheterization, probably because the tortuous outflow vessels stretch in accordance with the first microcatheter progress.

In conclusion, in a case of gastric varices in whom BRTO failed because of difficulty in advancing the microballoon catheter to the subphrenic branch vein, and in a case of tortuous and elongated GR shunt, DMRTO successfully achieved eradication of the varices. In both of these cases that were not eligible for BRTO, DMRTO became an alternative method of treatment.

\section{Disclosure Statement}

The authors have no financial disclosures to make. 

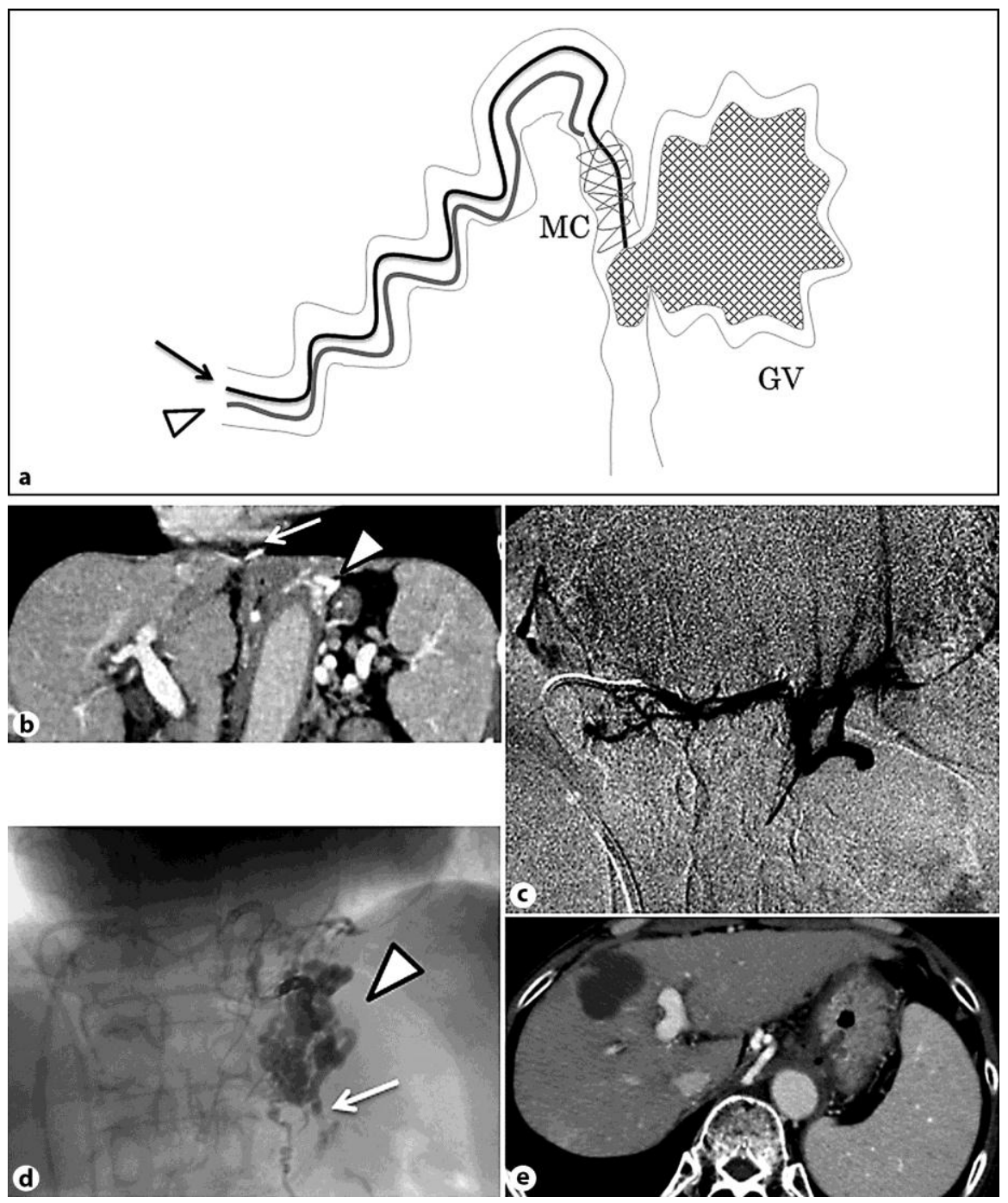

Fig. 1. a Diagram of DMRTO. The arrow and arrowhead indicate the two microcatheters. MC = Microcoils; GV = gastric varices. $\mathbf{b}$ Coronal view of dynamic contrast-enhanced CT showed the gastric varices (arrowhead) and the subphrenic vein (arrow) as the main draining vein. c Microballoon-occluded subphrenic venography through the guide catheter showed the subphrenic vein and phrenic branch vein, but the gastric varices were not visualized. $\mathbf{d}$ Gastric varices (arrowhead) and the inflow vessel (arrow) were revealed on gastric varicography. Dual microcatheters were inserted through the dual guide catheters, and contrast medium was injected via a microcatheter advanced to the gastric varices, following coil embolization of the draining vein through the second microcatheter. e Dynamic contrast-enhanced CT confirmed thrombosis of the gastric varices. 

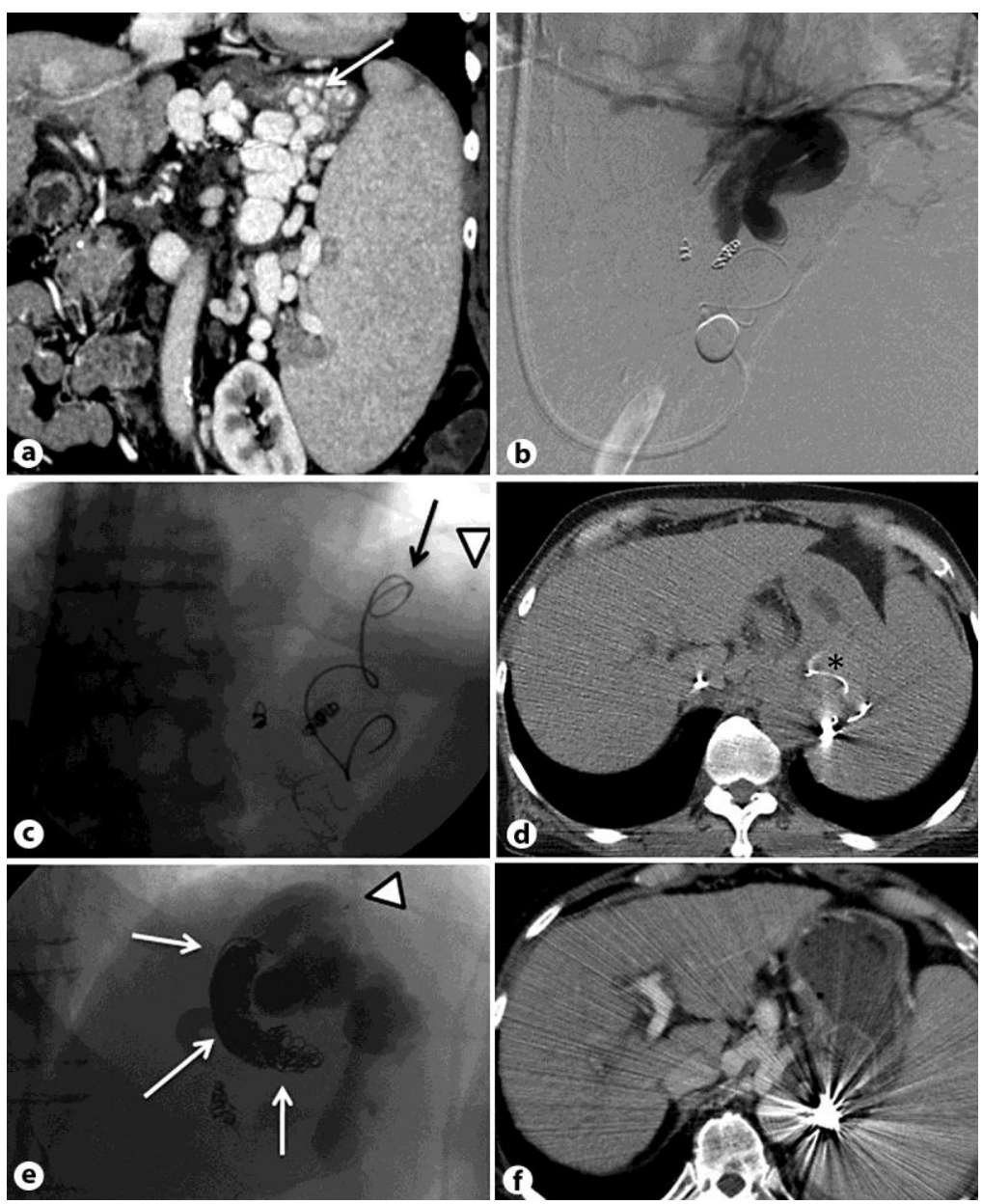

Fig. 2. a Coronal view of dynamic CT showed gastric varices (arrow) and numerous varicosities including inflow and draining vessels. b Balloon-occluded retrograde venography using a microcatheter under balloon inflation of the left adrenal vein following coil embolization of the small collateral outflows. The subphrenic, cardiophrenic, and pericardial vein were apparent but the gastric vein was not visualized. c Abdominal radiograph showed dual microcatheters inserted through dual guide catheters situated in the left adrenal vein. One microcatheter was advanced to a site adjacent to the gastric varices (arrowhead) and the other was advanced to several centimeters back from the gastric varices (arrow). $\mathbf{d}$ Angio-CT axial image just before the injection of sclerosant. The first microcatheter was adjacent to the gastric varices $(*)$. e In the right anterior oblique view, the gastric varices and the inflow vessel were injected with sclerosant through the first microcatheter (arrowhead) following coil embolization (arrows) of the outflow vessel through the second microcatheter. $f$ Dynamic contrast-enhanced CT showed no enhancement of the gastric varices. Microcoils placed in the outflow vessel were apparent. 


\section{References}

1 Kanagawa H, Mima S, Kouyama H, Gotoh K, Uchida T, Okuda K: Treatment of gastric fundal varices by balloon-occluded retrograde transvenous obliteration. J Gastroenterol Hepatol 1996;11:51-58.

$\checkmark 2$ Kiyosue H, Tanoue S, Kondo Y, et al: Balloon-occluded retrograde transvenous obliteration of complex gastric varices assisted by temporary balloon occlusion of the splenic artery. J Vasc Interv Radiol 2011;2:1045-1048.

-3 Maeda H, Hirota S, Yamamoto S, et al: Radiologic variations in gastrorenal shunts and collateral veins from gastric varices in images obtained before balloon-occluded retrograde transvenous obliteration. Cardiovasc Intervent Radiol 2007;30:410-414.

4 Kiyosue H, Mori H, Matsumoto S, Yamada Y, Hori Y, Okino Y: Transcatheter obliteration of gastric varices. Part 1. Anatomic classification. Radiographics 2003;23:911-920.

-5 Hirota S, Kobayashi K, Maeda H, et al: Balloon-occluded retrograde transvenous obliteration for portal hypertension. Radiat Med 2006;24:315-320.

-6 Wildrich WC, Srinivasan M, Semine MC, Robbins AH: Collateral pathways of the left gastric vein in portal hypertension. AJR Am J Roentgenol 1984;142:375-382.

$>7$ Nishida N, Ninoi T, Kitayama T, et al: Dual balloon-occluded retrograde transvenous obliteration of gastric varix draining into the left adrenal vein and left inferior phrenic vein. Cardiovasc Intervent Radiol 2004;27:560-562.

8 Kameda N, Higuchi K, Shiba M, et al: Management of gastric fundal varices without gastro-renal shunt in 15 patients. World J Gastroenterol 2008;14:448-453.

-9 Koito K, Namieno T, Nagakawa T, Morita K: Balloon-occluded retrograde transvenous obliteration for gastric varices with gastrorenal or gastrocaval collaterals. AJR Am J Roentgenol 1996;167:1317-1320.

10 Kiyosue H, Mori H, Matsumoto S, Yamada Y, Hori Y, Okino Y: Transcatheter obliteration of gastric varices. Part 2. Strategy and techniques based on hemodynamic features. Radiographics 2003;23: 921-937.

11 Yamagami T, Kato T, Hirota T, et al: Infusion of 50\% glucose solution before injection of ethanolamine oleate during balloon-occluded retrograde transvenous obliteration. Australas Radiol 2007;51:334-338.

12 Takahashi K, Yamada T, Hyodoh H, et al: Selective balloon-occluded retrograde sclerosis of gastric varices using a coaxial microcatheter system. AJR Am J Roentgenol 2001;177:1091-1093.

13 Sugimori K, Morimoto M, Shirato K, et al: Retrograde transvenous obliteration of gastric varices associated with large collateral veins or a large gastrorenal shunt. J Vasc Interv Radiol 2005;16:113-118.

14 Fukuda T, Hirota S, Sugimoto K, Matsumoto S, Zamora CA, Sugimura K: ‘Downgrading' of gastric varices with multiple collateral veins in balloon-occluded retrograde transvenous obliteration. J Vasc Interv Radiol 2005;16:1379-1383.

15 Tanoue S, Kiyosue H, Matsumoto S, et al: Development of a new coaxial balloon catheter system for balloon-occluded retrograde transvenous obliteration (BRTO). Cardiovasc Intervent Radiol 2006;29: 991-996. 